\title{
Synthesis of Amphiphilic Copolymers by Soap-free Interface-Mediated Polymerization
}

\author{
By Ryo KoJIMA, ${ }^{1,2}$ Maria Carmelita Z. KASUYA, ${ }^{1}$ Kazuhiko ISHIHARA, ${ }^{2}$ and Kenichi HATANAKA ${ }^{1, *}$
}

Amphiphilic copolymers were synthesized by soap-free heterogeneous polymerization and a novel method of polymer composition control for heterogeneous polymerization is proposed. Various copolymers of 2-methacryloyloxyethylphosphorylcholine (MPC) and alkyl methacrylates, such as $n$-hexyl methacrylate (HMA), $n$-dodecyl methacrylate (DMA), and stearyl methacrylate (SMA), were synthesized by soap-free heterogeneous polymerization. The reaction was carried out with the same monomer concentration but at different solvent compositions. This strategy could control the polymer composition despite the same monomer ratio. The behaviors of polymer composition were different depending on alkyl methacrylate species. It appears that a change of solvent polarity causes a change of the interface between the solution phase and the alkyl methacrylate phase. This phenomenon could be accounted on two factors. First, an increase in the solvent polarity results in an increase of the methacryloyl moieties which orient to the interface to solution phase because methacryloyl moieties are relatively more polar than alkyl chains. Second, the tendency of alkyl methacrylate phase to disperse in the solution phase decreases as the solvent polarity increases.

KEY WORDS: Amphiphilic Copolymer / Heterogeneous Polymerization / Polymer Composition Control / Interface / 2-Methacryloyloxyethylphosphorylcholine /

Various amphiphilic copolymers have been reported in the past since these copolymers have potential application to drug delivery system, biocompatible materials, and micro or nano spheres. $^{1-7}$ These amphiphilic copolymers have been synthesized by methods such as two-step polymerization, dispersion polymerization, ring-opening polymerization, and chemical modification after polymerization. ${ }^{5,8-12}$ However, these synthetic methods need additives like dispersant or require at least two step reactions. It is desired to synthesize amphiphilic copolymers by one step reaction without additives. Especially, it is industrially important. Soap-free heterogeneous polymerization which copolymerizes hydrophilic and hydrophobic monomers in two phases meets these requirements. There are several reports about soap-free heterogeneous polymerization. Zhang et al. copolymerized styrene and hydrophilic monomer, 2-methacrylic acid 3-bis-(carboxymethylamino)-2-hydroxypropyl ester, by soap-free heterogeneous polymerization and used it as scaffold to synthesize Pd nanoparticles. ${ }^{6}$ Similarly, Wen and co-workers synthesized microspheres, copolymer of hydrophobic and relatively hydrophilic monomers. ${ }^{7}$ However, they also reported that the same hydrophobic monomer and more hydrophilic monomer were not copolymerized under the same reaction system.

Herein, amphiphilic copolymers were synthesized by soapfree heterogeneous polymerization and a novel method of polymer composition control for heterogeneous polymerization is proposed. In spite of the same monomer ratio, this method could control polymer composition by changing only the solvent composition. We also discuss the mechanism of this method. Three kinds of alkyl methacrylates, $n$-hexyl meth- acrylate (HMA), n-dodecyl methacrylate (DMA), and stearyl methacrylate (SMA), were used as hydrophobic monomer and 2-methacryloyloxyethylphosphorylcholine (MPC) was chosen as hydrophilic monomer. MPC has a phosphorylcholine moiety and is known to be a biocompatible molecule. ${ }^{3}$ Therefore, these amphiphilic copolymers are also anticipated to have useful application for biomaterials.

\section{EXPERIMENTAL}

\section{Materials}

HMA, DMA, and SMA were purchased from Tokyo Chemical Industry and used as received. Ammonium persulfate (APS) and pyrene were supplied by Wako Pure Chemical industries and used without further purification. MPC was synthesized by a previously reported method. ${ }^{13}$ The structure of MPC is shown in Figure 1.

\section{Synthesis of Poly(alkyl methacrylate-co-MPC)}

$1.00 \mathrm{mmol}$ of MPC and $0.100 \mathrm{mmol}$ of APS were weighed in a flask and $20 \mathrm{~mL}$ of solvent and $1.00 \mathrm{mmol}$ of alkyl methacrylate were added. Water and dimethylsulfoxide (DMSO) were used as solvent and the ratio was varied. Then,

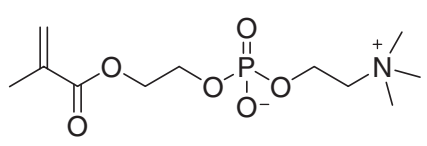

Figure 1. The structure of MPC.

\footnotetext{
${ }^{1}$ Institute of Industrial Science, The University of Tokyo, 4-6-1, Komaba, Meguro-ku, Tokyo, Japan

${ }^{2}$ Graduate School of Engineering, The University of Tokyo, 7-3-1, Hongo, Bunkyo-ku, Tokyo, Japan

*To whom correspondence should be addressed (Tel: +81-3-5452-6355, Fax: +81-3-5452-6356, E-mail: hatanaka@iis.u-tokyo.ac.jp).
} 
the mixture was degassed and the heterogeneous polymerization was initiated under Ar atmosphere at $60^{\circ} \mathrm{C}$ for $20 \mathrm{~h}$ (HMA or SMA) or $20 \mathrm{~min}$ (DMA). In order to form uniform oil droplets, stirring rate was kept at $1.00 \times 10^{3} \mathrm{rpm}$ and the same flask and magnetic stirrer bar were used for all experiments. After polymerization, the mixture was added to a large amount of acetone and filtered. Finally, the precipitate was dialyzed using a 3500 cut off membrane for $3 \mathrm{~d}$.

\section{Synthesis of MPC Homopolymer}

$1.00 \mathrm{mmol}$ of MPC and $0.100 \mathrm{mmol}$ of APS were dissolved in $10 \mathrm{~mL}$ of DMSO and $10 \mathrm{~mL}$ of water. Then, the mixture was degassed and polymerization was initiated under $\mathrm{Ar}$ atmosphere at $60^{\circ} \mathrm{C}$ for $20 \mathrm{~h}$. The product was purified as described above.

\section{Homogeneous Synthesis of Poly(HMA-co-MPC)}

As a reference, HMA and MPC were copolymerized homogeneously. $1.00 \mathrm{mmol}$ of MPC, $1.00 \mathrm{mmol}$ of HMA, and $0.020 \mathrm{mmol}$ of APS were dissolved in $1.5 \mathrm{~mL}$ of methanol and $0.5 \mathrm{~mL}$ of ethanol. Then, the mixture was degassed and polymerization was initiated under $\mathrm{Ar}$ atmosphere at $60^{\circ} \mathrm{C}$ for $20 \mathrm{~h}$. The product was purified as described above.

\section{Characterization}

It is difficult to determine the polymer composition by ${ }^{1} \mathrm{H}$ NMR since the peaks corresponding to MPC and alkyl methacrylate moieties overlap. Therefore, elemental analysis (Fisons Instruments EA-1108) was used to determine the mole fraction of the MPC moiety of the copolymers. The molecular weight of the polymer was estimated using size-exclusion chromatography (column, TOSOH TSK-gel G-Oligo-PW, $\mathrm{G} 2500 \mathrm{PW} \mathrm{XL}_{\mathrm{XL}}, \mathrm{G} 3000 \mathrm{PW} \mathrm{XL}_{\mathrm{X}}, \mathrm{G} 4000 \mathrm{PW} \mathrm{XL}_{\mathrm{XL}}$; standard, pullulan; eluent, water). The change of RI and UV absorption were measured. To determine the critical micelle concentration (CMC) of copolymers, the fluorescence shift of pyrene was examined by fluorescence spectrophotometer (Hitachi F-2500). The viscosity of alkyl methacrylate was measured using a viscometer (A\&D SV-1A).

\section{RESULTS AND DISCUSSION}

Figure 2 shows the relationship between the reaction solvent composition and the polymer composition. The polymer composition clearly changed by varying the reaction solvent composition in spite of the same monomer ratio in feed. The alkyl methacrylate content of poly(HMA-co-MPC) increased and remained constant as the water content of solvent increased. HMA and MPC were copolymerized even in a large amount of water. On the other hand, the alkyl methacrylate content of poly(DMA-co-MPC) decreased with an increase of the water content of solvent. Only MPC was polymerized in a large amount of water. The alkyl methacrylate content of poly(SMAco-MPC) behaved similarly to poly(DMA-co-MPC).

Figure 3 shows the size-exclusion chromatogram. Solid line and dotted line refer to the change of RI and UV absorption,

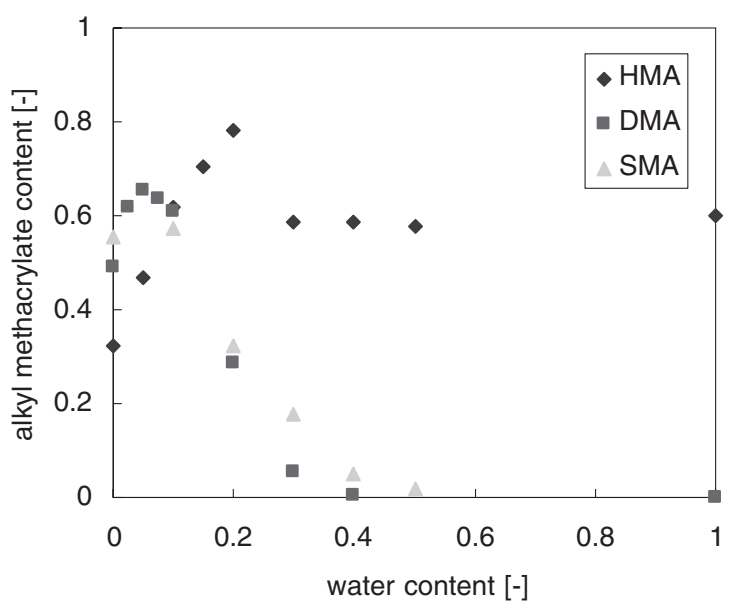

Figure 2. The relationship between polymer composition and reaction solvent composition.

respectively. The chromatogram showed several peaks although the sample was synthesized by one-pot polymerization. Strong UV absorption was observed from the copolymer of high molecular weight. In contrast, UV absorption of MPC homopolymer was very weak. Hence, results showed that the molecular weight of the copolymer was proportional to its alkyl methacrylate content. Apparently, the products were mixtures of "high-molecular-weight alkyl-methacrylate-rich copolymer" and "low-molecular-weight MPC-rich copolymer."

In order to determine the CMC of copolymers, fluorescence shift of pyrene was examined. Pyrene is known that the wavelength of maximum absorption shifts from $334 \mathrm{~nm}$ in hydrophilic environment to $337 \mathrm{~nm}$ in hydrophobic environment. ${ }^{14}$ Consequently, poly(HMA-co-MPC) was dissolved in saturated pyrene solution at different concentration ranging from $10^{-5}$ to $1 \mathrm{mg} / \mathrm{mL}$ and absorption spectra were measured. As a reference, the samples of poly(HMA-co-MPC) which was synthesized homogeneously $\left(10^{-4} \sim 1 \mathrm{mg} / \mathrm{mL}\right)$ were also measured. The copolymer composition, $M_{\mathrm{n}}$, and $M_{\mathrm{w}}$ of these copolymers are shown in Table I. The ratio of fluorescence intensity $\left(\mathrm{I}_{337} / \mathrm{I}_{334}\right)$ was plotted versus the logarithm of polymer concentration (Figure 4). The CMC of heterogeneously synthesized poly(HMA-co-MPC) and homogeneously synthesized copolymer were $7.1 \times 10^{-4} \mathrm{mg} / \mathrm{mL}$ and $1.4 \times$ $10^{-3} \mathrm{mg} / \mathrm{mL}$, respectively. That is to say, heterogeneously synthesized poly(HMA-co-MPC) forms micelle more easily than homogeneously synthesized one. These results indicate that heterogeneously synthesized poly(HMA-co-MPC) has a block-like structure.

The model of heterogeneous polymerization accounts for these results (Figure 5). Alkyl methacrylate hardly dissolves in water and DMSO. Therefore, the mechanism for this polymerization could be inferred of as shown in Figure 5. To begin with, MPC is polymerized because the initiator of APS is soluble in the solvent. Then, there are two possibilities. One is that the polymerization reaction is terminated and MPC rich copolymer is obtained. The other is that the growing end of MPC oligomer reacts with the alkyl methacrylate molecule 
A

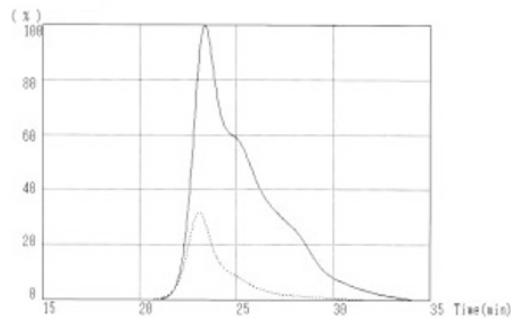

D

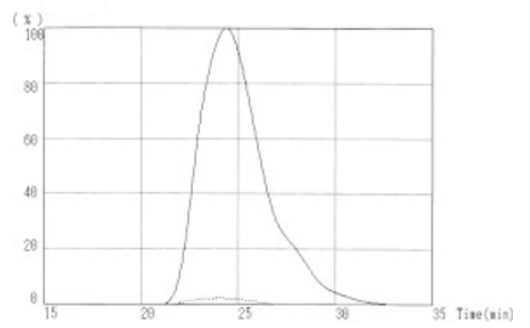

B

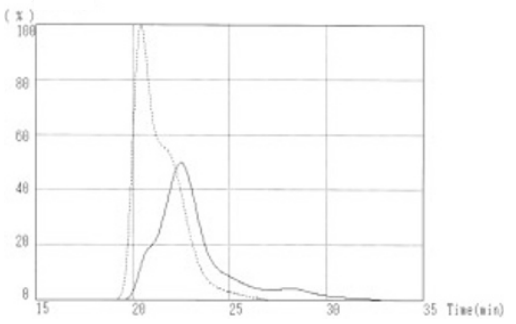

E

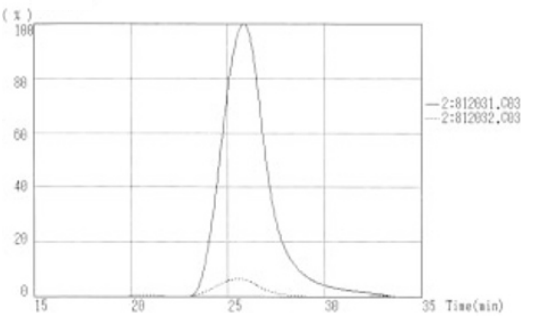

C

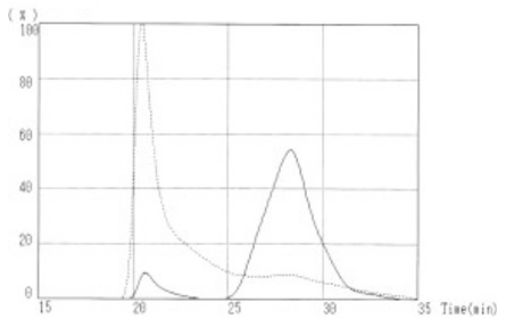

Figure 3. The size-exclusion chromatogram for (A) poly(HMA-co-MPC) (HMA:MPC = 62:38), (B) poly(DMA-co-MPC) (DMA:MPC = 61:39), (C) poly(SMA-coMPC) (SMA:MPC = 57:43), (D) MPC homopolymer, and (E) Homogeneously synthesized poly(HMA-co-MPC) (HMA:MPC = 56:44). The conditions were; column, TOSOH TSK-gel G-Oligo-PW, G2500PW $\mathrm{XL}_{\mathrm{L}}$, G3000PW $\mathrm{XL}_{\mathrm{L}}, \mathrm{G} 4000 \mathrm{PW} \mathrm{XL}_{\mathrm{XL}}$; standard, pullulan; eluent, water; sample concentration, $1 \%$ $(\mathrm{w} / \mathrm{w})$. Solid line and dotted line refer to the change of RI and UV absorption, respectively.

Table I. The copolymer composition and average molecular weight of heterogeneously and homogeneously synthesized poly(HMA-co-MPC). Heterogeneously synthesized copolymer was polymerized in $\mathrm{H}_{2} \mathrm{O}$ :DMSO $(1: 9 \mathrm{v} / \mathrm{v})$. The average molecular weight was estimated from the chromatogram of Figure 3. SEC conditions were same as that of Figure 3

\begin{tabular}{cccc}
\hline Synthetic method & HMA:MPC & $M_{\mathrm{n}}$ & $M_{\mathrm{w}}$ \\
\hline Heterogeneous & $62: 38$ & $1.90 \times 10^{4}$ & $7.53 \times 10^{4}$ \\
Homogeneous & $56: 44$ & $1.89 \times 10^{4}$ & $3.24 \times 10^{4}$ \\
\hline
\end{tabular}

which is at the interface between the solution phase and the alkyl methacrylate phase. In the latter case, it is anticipated that alkyl methacrylate molecules can easily polymerized since alkyl methacylate molecules are at high density in the alkyl methacrylate phase. Hence, high molecular weight copolymers are produced, presumably. In addition, it is expected that the products have block-like structure, in this case. This is the reason why the products were mixtures of "high-molecularweight alkyl-methacrylate-rich copolymer" and "low-molecular-weight MPC-rich copolymer.”

The alkyl methacrylate content of copolymers probably depends on the number of alkyl methacrylate molecules of which the active sites are at the interfaces between the solution phase and the alkyl methacrylate phase. This is because only the active sites of alkyl methacrylate molecules at the interfaces may be involved with copolymerization. It would appear that there are two factors which are concerned with the solvent polarity and the number of active sites of alkyl methacrylate molecules at the interfaces. First, an increase in the solvent polarity results in increasing the number of active sites which orient to the solution phase because methacryloyl moieties are relatively more polar than alkyl chains. Second,

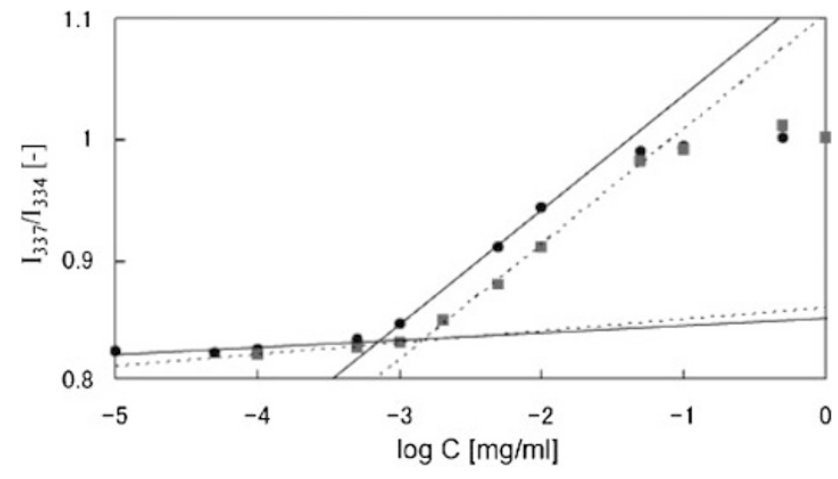

Figure 4. The change of $I_{337} / I_{334}$ monitored at $\lambda_{\mathrm{em}} 392 \mathrm{~nm}$ as a function of concentration of heterogeneously synthesized poly(HMA-co-MPC) (circle) with corresponding fitted line (solid line) and homogeneously synthesized poly(HMA-co-MPC) (square) with corresponding fitted line (dotted line).

the tendency of alkyl methacrylate phase to disperse in the solution phase decreases as solvent polarity increases. Thus, an increase of the solvent polarity enlarges the size of alkyl methacrylate droplet and decreases the surface area of the interfaces. It means a decrease of the number of active sites at the interfaces. The influence of alkyl methacrylate slightly dissolved in the solution phase is thought of as another factor. However, if this factor is dominant over the first and second factors, MPC rich copolymer will be produced in the homogenous solution phase by typical copolymerization. Therefore, this factor is probably less influential than the first and second factors.

As for the first factor, the alkyl methacrylate content of copolymers may increase with increasing the solvent polarity. 


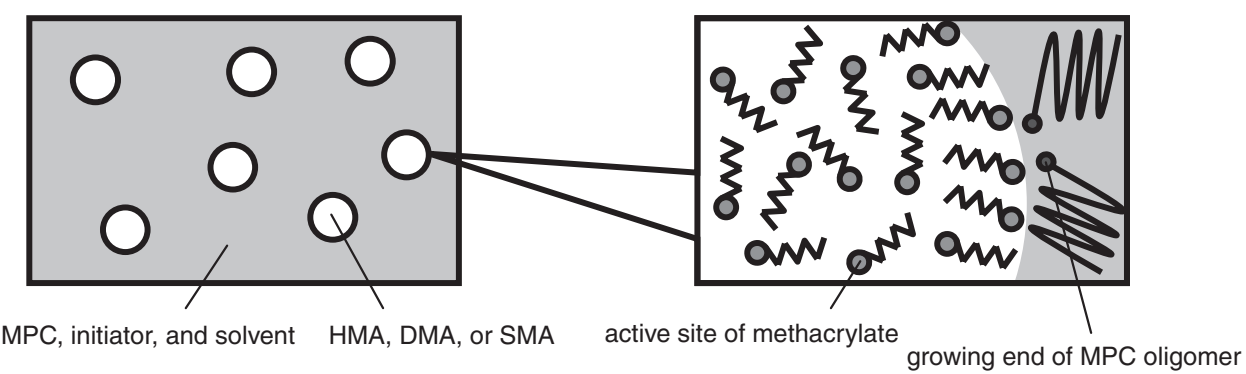

Figure 5. The model of heterogeneous polymerization

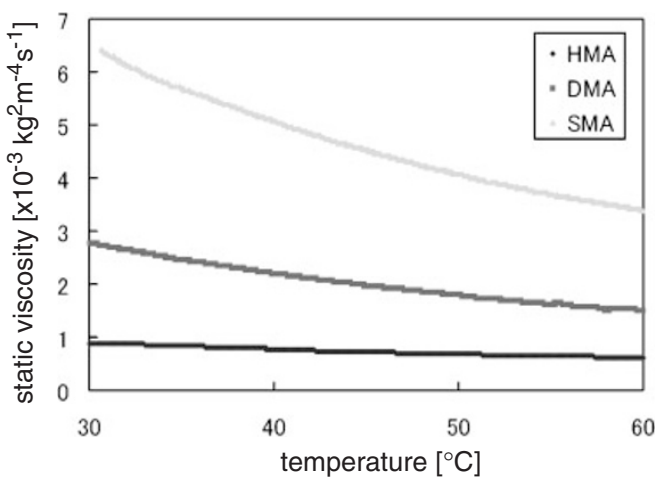

Figure 6. The static viscosity of alkyl methacrylate as a function of temperature. Liquid alkyl methacrylate was measured directly.

In contrast, as for the second factor, the alkyl methacrylate content of copolymers may decrease with an increase of the solvent polarity. It is anticipated that molecular motions of DMA and SMA are slow since these alkyl chains are long. Hence, DMA and SMA are less influenced by the first factor than by the second factor. Actually, the DMA and SMA contents of these copolymers decreased monotonically as the solvent polarity increased ranging from 10 to $100 \%$ of water content. Meanwhile, alkyl chain of HMA is short and molecular motion of HMA is fast, presumably. Therefore, the HMA content of the copolymer must be influenced by both factors. The methacrylate content of poly(HMA-co-MPC) neither increased nor decreased monotonically as the solvent polarity increased.

The static viscosity of alkyl methacrylate was measured to validate that the longer the alkyl chain of alkyl methacrylate becomes, the slower the molecular motion becomes (Figure 6). Viscosity is related to molecular interaction. ${ }^{15}$ At $60^{\circ} \mathrm{C}$, the static viscosities of HMA, DMA, and SMA were $0.63 \times 10^{-3}$, $1.50 \times 10^{-3}, \quad 3.40 \times 10^{-3} \mathrm{~kg}^{2} \mathrm{~m}^{-4} \mathrm{~s}^{-1}$, respectively. These results indicate that the molecular motion of alkyl methacrylate becomes slow as the alkyl chain elongates.

\section{CONCLUSION}

Amphiphilic copolymers of MPC and alkyl methacrylates were synthesized by soap-free heterogeneous polymerization.
The polymer composition could be controlled by varying the solvent composition because the change of solvent might causes the change of interface. These results suggest that the interface is critically important in heterogeneous polymerization. The condition and the surface area of the interface greatly influence copolymerization.

Acknowledgment. This work was supported by a grant for "Development of Novel Diagnostic and Medical Applications through Elucidation of Sugar Chain Functions" from the New Energy and Industrial Technology Development Organization (NEDO).

Received: December 17, 2008

Accepted: January 19, 2009

Published: March 4, 2009

\section{REFERENCES}

1. K. Kataoka, G. S. Kwon, M. Yokoyama, T. Okano, and Y. Sakurai, J. Controlled Release, 24, 119 (1993).

2. Y. Bae, S. Fukushima, A. Harada, and K. Kataoka, Angew. Chem., Int. Ed., 42, 4640 (2003).

3. T. Ueda, H. Oshida, K. Kurita, K. Ishihara, and N. Nakabayashi, Polym. J., 24, 1259 (1992).

4. K. Sugiyama, S. Mitsuno, and K. Shiraishi, J. Polym. Sci., Part A: Polym. Chem., 35, 3349 (1997).

5. W. Yang, D. Yang, J. Hu, C. Wang, and S. Fu, J. Polym. Sci., Part A. Polym. Chem., 39, 555 (2001).

6. M. Zhang and W. Zhang, J. Phys. Chem. C, 112, 6245 (2008).

7. F. Wen, W. Zhang, P. Zheng, X. Zhang, X. Yang, Y. Wang, X. Jiang, G. Wei, and L. Shi, J. Polym. Sci., Part A: Polym. Chem., 46, 1192 (2008).

8. C. J. Ferguson, R. J. Hughes, D. Nguyen, B. T. T. Pham, R. G. Gilbert, A. K. Serelis, C. H. Such, and B. S. Hawkett, Macromolecules, 38, 2191 (2005).

9. S. Xu and W. Liu, J. Fluorine. Chem., 129, 125 (2008).

10. D. Sarkar, J. M. E. Khoury, S. T. Lopina, and J. Hu, J. Appl. Polym. Sci., 104, 1905 (2007).

11. T. Kotre, O. Nuyken, and R. Weberskirch, Macromol. Rapid Commun., 23, 871 (2002).

12. K. Nakahama, H. Kawaguchi, and K. Fujimoto, Langmuir, 16, 7882 (2000).

13. K. Ishihara, T. Ueda, and N. Nakabayashi, Polym. J., 22, 355 (1990).

14. T. Suda, Y. Chida, H. Oku, K. Yamada, and R. Katakai, Presented at the 42nd Japanese Peptide Symposium, Senri Life Science Center, Osaka, Japan, October 27-29, 2005.

15. V. I. Deulin, Makromol. Chem., 180, 263 (1979). 